\section{Neuropsychobiology}

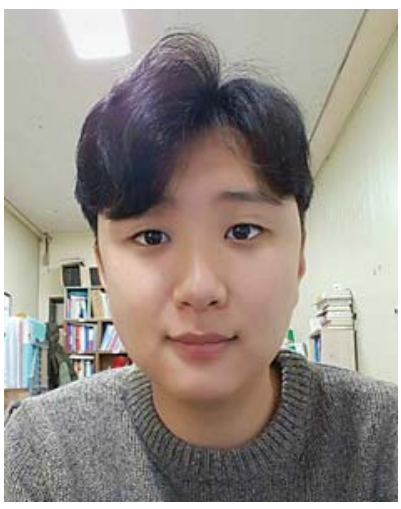

MinCheol Shin

\section{Abstracts}

Neuropsychobiology 2016;74:84-86

Published online: February 9, 2017

\title{
Abstracts of Award-Winning Posters, 16th International Forum on Mood and Anxiety Disorders, Rome, December 8-10, 2016
}

\author{
1 \\ A Study for Development of the Korean Version of the Beck \\ Hopelessness Scale in Sign Language \\ Hyejeong Jeong, Soontaeg Hwang, Hyeseon Jo, MinCheol Shin \\ Department of Psychology, Chungbuk National University, Cheongju, South Korea
}

Hopelessness is a noted variable in predicting suicide risk as a feature of depression. Deaf persons with impaired auditory function during the language development period experience difficulties in interpersonal relationships because of restrictions of communication and have affective vulnerability. Since deaf persons have difficulty in reading and understanding texts, there is a need to perform psychological examinations using sign language (Korean sign language $[\mathrm{KSL}]$ ) for them to understand the examination more easily. However, there are few psychological assessment tools to evaluate the effects. This study developed a sign language text version of the Korean version of the Beck Hopelessness Scale (K-BHS) for deaf persons and a sign language video version of the K-BHS. The original version of the K-BHS and the sign language text version of the K-BHS were completed by 50 hearing persons to verify reliability and validity. The video version of the KBHS was conducted for 227 deaf persons and test-retest was performed for 16 deaf persons. The text and video versions of the K-BHS was conducted by 24 examiners who were bilingual in Korean and KSL. For identifying relationships between hopelessness and depression in deaf persons, the original Korean version of the Beck Depression Inventory II (K-BDI-II) and the sign language video version of the K-BDI-II (K-BDI-II-KSL) were carried out for hearing persons, deaf persons, and bilingual persons. Cronbach's $\alpha$ of the original and sign language text versions of the K-BHS was 0.897 and 0.854 , and Cohen's $d$ between the original and sign language text versions was 0.139 . The correlation between the original and sign language text versions of the K-BHS was statistically significant. In addition, the correlation between the original and video versions of the K-BHS was significant. Cronbach's $\alpha$ of the video version of the K-BHS was 0.723 , and test-retest reliability was significant. Deaf persons showed significantly higher levels of hopelessness and depression than hearing persons. Additionally, the results showed that correlations between the K-BHS and K-BDI-II of the original version in hearing persons were higher than correlations between K-BHS and K-BDI-II of the video version in deaf persons. In summary, this study developed a sign language version of the K-BHS for deaf persons, tested its reliability and validity, and identified levels of hopelessness and depression among deaf persons. 


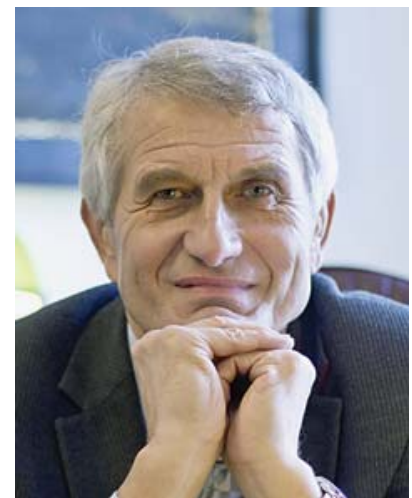

Jiri Raboch

\section{Mitochondrial Respiration in Bipolar Affective Disorder}

Jiri Raboch ${ }^{a}$, Jana Hroudováa , Ekaterina Sigitova ${ }^{a}$, Hana Hansíkováb, Zdeněk Fišar $^{a}$, Lucie Kalisova ${ }^{a}$, Eva Kitzlerova ${ }^{a}$, Martina Zvěřováa

Departments of apsychiatry and bediatrics, Charles University, 1st Faculty of Medicine, Prague, Czech Republic

Bipolar affective disorder $(\mathrm{BAD})$ is a serious mental disorder. The predisposition to $\mathrm{BAD}$ is determined by genetic and other biological and psychosocial factors. Changes in the activities of compounds of intracellular signaling pathways are studied with the aim of discovering new biological markers of mood disorders or predictors of response to pharmacotherapy, which can be easily examined in blood samples.

The aim of our study was to determine whether there is an association between changes in energy metabolism and different episodes of BAD. Selected mitochondrial parameters were measured in peripheral blood components. The analyses were examined in BAD patients of varying states and in healthy controls. Clinical evaluation of the BAD patients was provided by experienced clinicians using the following questionnaires: MDQ, MADRS, YMRS, CGI-S, and BPRS. Mitochondrial respiration was examined in intact and permeabilized blood platelets using high-resolution respirometry. It was evaluated by both respiratory rate and respiratory control ratios. Enzyme activities (citrate synthase, electron transport chain complexes - complex I, II+III, IV) were measured spectrophotometrically. Statistical analyses were performed using the STATISTICA data analysis software system, version 12. Activities of individual complexes were normalized to citrate synthase activity. Statistical significance was evaluated using ANOVA and a post hoc Scheffé test.

Our preliminary results showed increased physiological respiration in intact platelets from manic patients. Complex-I linked respiration was found to be increased in manic patients and in remission compared to healthy controls. Citrate synthase activity was not changed in BAD patients compared to controls. Decreased complex IV activity was observed in $\mathrm{BAD}$ depressive patients in comparison to controls.

Our results support the hypothesis that changes in the rate of oxygen consumption and activities of electron transport chain complexes may participate in the pathophysiology of BAD. In conclusion, better insight into molecular mechanisms of cellular respiration could lead to a better understanding of the pathophysiology of BAD. Mitochondrial dysfunctions in different episodes of BAD should be further studied.

This study was supported by grants MZd ČR 15-28616A, P26/LF1/4, and PO3/LF1/9.

\section{3 \\ Are Inflammation and Cognition Related in Major Depression? Inadvertent Selection for a Cognitively Unimpaired Subgroup}

Smadar Valérie Tourjman ${ }^{a}$, Emmanuel Stip ${ }^{b}$, Glenda McQueen $^{c}$, Stéphane Potvin ${ }^{a}$, Miriam Beauchamp ${ }^{d}$, Edouard Kouassie

aDepartment of Psychiatry, Faculty of Medicine, University of Montreal, Institut Universitaire en Santé Mentale de Montréal, Montreal, QC, 'b Department of Psychiatry, Faculty of Medicine, University of Montreal, Montreal, QC, 'Department of Psychiatry, Faculty of Medicine, University of Calgary, Calgary, AB, dDepartment of Psychology, University of Montreal, Montreal, QC, and eDepartment of Medicine, Faculty of Medicine, University of Montreal, Montreal, QC, Canada

Deficits in cognition are frequent in major depression and may contribute to the decrements in functioning that are associated with depression $[1,2]$. The pathophysiological status of this symptom dimension in depression remains unclear. It is possible that cognitive deficit may be a trait, a state-related dimension, or even a "scar" resulting from untreated or chronic depression [3]. A recent study suggested that remitted first episode 
patients performed worse than those with remitted recurrent depression [4], while a meta-analysis showed cognitive deficits to be present as of the first episode [5]. However, another study showed better functioning in patients with a first episode of major depression [6]. During the course of 2 studies seeking to examine the relationship between cognitive function and inflammatory factors, we recruited subjects with a major depressive episode with onset occurring within the last 12 months. Further, inflammatory illnesses, unstable medical conditions, or any condition requiring the use of medication with antiinflammatory effects were exclusionary criteria. We present the cognitive profile of this sample, as well as the response to unblinded treatment with antidepressants. The majority of the sample was free of cognitive deficits and the response rates were unexpectedly high. We speculate that the selection criteria may have characterized a subpopulation of individuals with major depression who are as yet "unscarred" by the pathological process.

\section{References}

1 Bortolato B, et al: Cognitive remission: a novel objective for the treatment of major depression? BMC Med 2016;22:14.

2 Papakostas GI: Antidepressants and their effect on cognition in major depressive disorder. J Clin Psychiatry 2015;76:e1046.

3 Allott K, et al: Characterizing neurocognitive impairment in young people with major depression: state, trait, or scar? Brain Behav 2016;6:e00527.

4 Roca M, López-Navarro E, Monzón S, Vives M, García-Toro M, García-Campayo J, Harrison J, Gili $\mathrm{M}$ : Cognitive impairment in remitted and non-remitted depressive patients: a follow-up comparison between first and recurrent episodes. Eur Neuropsychopharmacol 2015;25:1991-1998.

5 Lee RS, et al: A meta-analysis of cognitive deficits in first-episode major depressive disorder. J Affect Disord 2012;140:113-124.

6 Talarowska M, et al: Cognitive functions in first-episode depression and recurrent depressive disorder. Psychiatr Danub 2015;27:38-43. 